\title{
Trait Specific Expression Profiling of Salt Stress Responsive Genes in Diverse Rice Genotypes as Determined by Modified Significance Analysis of Microarrays
}

\author{
Mohammad R. Hossain ${ }^{1,2 *}$, George W. Bassel ${ }^{2}$, Jeremy Pritchard ${ }^{2}$, Garima P. Sharma ${ }^{2}$ \\ and Brian V. Ford-Lloyd ${ }^{2}$ \\ ${ }^{1}$ Department of Genetics and Plant Breeding, Bangladesh Agricultural University, Mymensingh, Bangladesh, ${ }^{2}$ School of \\ Biosciences, University of Birmingham, Birmingham, UK
}

\section{OPEN ACCESS}

Edited by:

Shabir Hussain Wani,

Sher-e-Kashmir University of Agricultural Sciences \& Technology of

Kashmir, India

Reviewed by:

Taras P. Pasternak,

Institut für Biologie II, Germany

Tofazzal Islam,

Bangabandhu Sheikh Mujibur Rahman

Agricultural University, Bangladesh

*Correspondence: Mohammad R. Hossain

m.r.hossain@bau.edu.bd

Specialty section:

This article was submitted to

Plant Biotechnology,

a section of the journa

Frontiers in Plant Science

Received: 29 December 2015 Accepted: 12 April 2016

Published: 03 May 2016

Citation:

Hossain MR, Bassel GW, Pritchard J, Sharma GP and Ford-Lloyd BV (2016)

Trait Specific Expression Profiling of

Salt Stress Responsive Genes in

Diverse Rice Genotypes as

Determined by Modified Significance Analysis of Microarrays.

Front. Plant Sci. 7:567.

doi: 10.3389/fpls.2016.00567
Stress responsive gene expression is commonly profiled in a comparative manner involving different stress conditions or genotypes with contrasting reputation of tolerance/resistance. In contrast, this research exploited a wide natural variation in terms of taxonomy, origin and salt sensitivity in eight genotypes of rice to identify the trait specific patterns of gene expression under salt stress. Genome wide transcptomic responses were interrogated by the weighted continuous morpho-physiological trait responses using modified Significance Analysis of Microarrays. More number of genes was found to be differentially expressed under salt stressed compared to that of under unstressed conditions. Higher numbers of genes were observed to be differentially expressed for the traits shoot $\mathrm{Na}^{+} / \mathrm{K}^{+}$, shoot $\mathrm{Na}^{+}$, root $\mathrm{K}^{+}$, biomass and shoot $\mathrm{Cl}^{-}$, respectively. The results identified around 60 genes to be involved in $\mathrm{Na}^{+}, \mathrm{K}^{+}$, and anion homeostasis, transport, and transmembrane activity under stressed conditions. Gene Ontology (GO) enrichment analysis identified $1.36 \%$ (578 genes) of the entire transcriptome to be involved in the major molecular functions such as signal transduction (>150 genes), transcription factor (81 genes), and translation factor activity (62 genes) etc., under salt stress. Chromosomal mapping of the genes suggests that majority of the genes are located on chromosomes 1, 2, 3, 6, and 7. The gene network analysis showed that the transcription factors and translation initiation factors formed the major gene networks and are mostly active in nucleus, cytoplasm and mitochondria whereas the membrane and vesicle bound proteins formed a secondary network active in plasma membrane and vacuoles. The novel genes and the genes with unknown functions thus identified provide picture of a synergistic salinity response representing the potentially fundamental mechanisms that are active in the wide natural genetic background of rice and will be of greater use once their roles are functionally verified.

Keywords: transcriptomics, significance analysis of microarrays (SAM), rice, salt tolerance, gene ontology enrichment, regulatory network 


\section{INTRODUCTION}

Abiotic stresses greatly reduce agricultural productivity worldwide. The yield of rice, one of the major food crops that feed the world, can be reduced by up to 50\% making it highly sensitive to soil salinity. Salinity causes accumulation of excess $\mathrm{Na}^{+}$and $\mathrm{Cl}^{-}$in the shoot which is detrimental for plant nutrition and exerts osmotic stress and ionic toxicity that ultimately restricts plant growth (Munns et al., 2006; Flowers and Colmer, 2008; Munns and Tester, 2008). Salinity tolerance, a complex trait both physiologically and genetically, requires a wide range of physiological and biochemical responses by activating a complex network of genes upon exposure to stress (Cotsaftis et al., 2011; Wu et al., 2013). However, the naturally occurring genetic variation across rice varieties, cultivars, landraces and wild species provides the advantage to identify factors such as genes, proteins and metabolites which can be utilized by conventional breeding and genetic engineering technologies for improvement of crops' tolerance to stresses (Langridge and Fleury, 2011; Horie and Karahara, 2012).

The advances in the high throughput multi-omics techniques along with the progress made in the ever spreading arena of bioinformatics, have given rise to the system biology approaches (Duque et al., 2013). This allows the investigation of the natural genotypic variation holistically to gain deeper biological insight on how the plant functions as a whole by discovering the putative functions of genes, proteins and metabolites in a specific biological context by dissecting the complex regulatory networks of genes, proteins, and metabolites associated with stress adaptation and tolerance (Mochida and Shinozaki, 2011; Shelden and Roessner, 2013).

Recently, there has been a substantial advancements in the technology of whole genome transcriptomic profiling and a number of crop species such as Arabidopsis (Kumari et al., 2008), barley (Close and Wanamaker, 2004), maize (Wang et al., 2003), and wheat (Clarke and Rahman, 2005) etc., have been studied extensively. In rice, several microarray platforms were used such as cDNA microarrays (Ueda et al., 2006), NSF $45 \mathrm{~K}$ 70-mer oligo microarrays (Senadheera et al., 2009), Affymetrix gene chips (Walia et al., 2005; Walia and Wilson, 2007, 2009; Cotsaftis et al., 2011) and Agilent $44 \mathrm{k}$ microarray (Aya et al., 2011) to study the response of plants toward stresses at transcritpome level.

Stress responsive transcripts, in most of the cases, are usually identified based on comparative and differential transcriptomic expression analysis in two to four genotypes showing extremely contrasting levels of tolerance which results in the findings being genotype specific. This ceases the opportunity to analyze the gradient of transcriptomic responses in diverse genotypes at a time and to correlate those with the changes in morphophysiological responses to identify the significant transcripts and hence, the molecular functions and biological processes that are operating in species level as adaptive mechanisms (Feder and Walser, 2005; Hossain et al., 2015b). The recent advent of powerful and holistic analytical approaches of system biology such as the Significance Analysis of Microarrays (SAM) offer unique possibility in this regard. And such approaches of correlating the transcriptomic and morpho-physiological responses in a wide genetic background of rice under salt stress are yet to be explored.

SAM was first successfully used to identify the significant changes in transcriptional responses in human lymphoblastoid cells under ionizing radiation with a much lower estimated FDR (12\%), compared to the higher FDRs $(60-84 \%)$ of other conventional methods of analysis (Tusher et al., 2001). This study used a slightly modified version of SAM to suit it with the objective of analyzing the gradient of salinity induced transcriptomic and morpho-physiological responses in diverse genotypes of rice. To achieve this diversity, eight rice genotypes were used that are of diverse geographical origins and belong to different sub-species groups (such as indica, japonica, and wild species), different cultivar groups (such as landraces, cultivars, and high yielding varieties) with a reputation of different levels of tolerance to salinity stress (such as susceptible, moderately tolerant, and highly tolerant). Agilent's $44 \mathrm{k}$ oligo-microarrays were used as it provided less expensive yet reasonably sensitive profiling of the genome-wide transcriptional responses of such higher number of samples.

Thus, a modified SAM approach is used to interrogate the patterns of variation in gene expressions by the gradient of responses in morpho-physiological traits in a wide genetic background of rice represented by eight diverse (in terms of taxonomy, origin and salt sensitivity) genotypes in a way to identify the significant transcripts that is relative to the changes in a particular morpho-physiological trait under salt stress. These identified trait specific salinity induced transcripts are discussed along with their complex regulatory networks and the major biological processes and molecular functions that are operating in the wide genetic background of rice as adaptive mechanisms to cope with the stressed environments.

\section{MATERIALS AND METHODS}

\section{Plant Materials}

Eight rice genotypes (Table 1) consisting of landraces, cultivars, high yielding varieties (HYVs) and wild species and taxonomically belonging to three different rice sub-species groups namely, Indica, Japonica, and wild species, having diverse geographical origin and showing different levels of tolerance to salt stress were obtained from the International Rice Gene Bank Centre (IRGC) of the International Rice Research Institute (IRRI).

\section{Hydroponic Culture Conditions and Salinity Treatments}

Plants were grown twice maintaining the same environmental conditions in growth room. In the first growing, the seedlings in the flasks were challenged with $80 \mathrm{mM} \mathrm{NaCl}$ at 14 days after seedling emergence (DAE) and the data for the 14 morphophysiological traits were collected within the next 7 days. The details of the traits (names of the traits are shown in Figure 1) can be found in Hossain et al. (2015a). In the second growing, $120 \mathrm{mM} \mathrm{NaCl}$ stress was used and total RNA from the whole seedlings was extracted after $48 \mathrm{~h}$ of stressed period (i.e., at 16 DAE) for whole genome transcriptome profiling. The details 
TABLE 1 | List of diverse rice genotypes along with their sup-species levels, origins, and reputation of salt stress used for trait specific expression profiling of salinity induced transcripts by modified Significance Analysis of Microarrays (SAM).

\begin{tabular}{|c|c|c|c|c|}
\hline Genotype & Germplasm Group & Accession Number & Origin & Salt Tolerance status (Hossain et al., 2015a) \\
\hline Pokkali & Indica (landrace) & IRGC 108921 & India & Tolerant \\
\hline PSBRc50 & Indica (variety) & IRGC 99706 & Philippines & Moderately tolerant \\
\hline IR 58 & Indica (variety) & IRGC 63492 & Philippines & Moderately tolerant \\
\hline BRRI dhan 29 & Indica (HYV) & IRTP 15241 & Bangladesh & Susceptible \\
\hline Banikat & Japonica (cultivar) & IRGC 67720 & India & Moderately tolerant \\
\hline Nipponbare & Japonica (cultivar) & IRGC 117274 & Japan & Susceptible \\
\hline O. latifolia & Wild species & IRGC 100965 & Costa Rica & Susceptible \\
\hline O. rufipogon & Wild species & IRGC 105390 & Thailand & Susceptible \\
\hline
\end{tabular}

HW, High yielding variety, IRGC, International Rice Germplasm Center, IRTP, International Rice Testing Program.

of the plant growth conditions, salinity treatments, and the collection of morpho-physiological data can be found in Hossain et al. (2015a).

\section{Extraction and Quality Assessment of Total RNA}

The total RNA was extracted from the 16 days old seedlings using the QIAGEN RNeasy Plant Mini Kit. For seedlings weighing more than the recommended amount of starting material (100 $\mathrm{mg}$ ), the sections of root, stem, and leaves were used to represent the whole seedlings. Each sample from each individual seedling represented a replicate. The freshly harvested samples were snap frozen in liquid nitrogen and were immediately ground to a fine powder with pre-chilled mortar and pestle. The RNA was purified using an RNeasy spin column and an on-column DNase treatment. The total RNA was eluted in $30 \mu \mathrm{l}$ of RNase free water and was immediately aliquoted and stored in a $-70^{\circ} \mathrm{C}$ freezer. Only the eluted total RNA having the 260/280 and 260/230 ratios around 2.0 (as quantified by Nanodrop ND1000 VIS spectrophotometer v. 3.2.1) and having the RNA integrity (RIN) value $>7.0$ (as detected by the Agilent 2100 bioanalyser using the RNA 6000 Nano Labchip kit) were used hybridization.

\section{Microarray Hybridization, Feature Extraction, and Rice Genome Array}

The 48 extracted RNA samples were then prepared for microarray hybridization in the Genomics Services Facility of the University of Birmingham where cDNA synthesis, preparation of One-Color Spike-Mix, Cy3 labeling, microarray hybridization on Agilent $4 \times 44 \mathrm{~K}$ rice oligo microarrays, scanning and feature extraction was carried out using Agilent's one color Quick Amp labeling kit (v.6.5, May 2010). The array represented about 43,803 rice genes with one 60 -mer oligonucleotide probe representing each and consists of 32,325 probes corresponding to RAP loci with full-length representative cDNA, 6943 probes to RAP loci with EST support, 2612 probes to gene predicted loci and control probes including false positives and non-rice sequences etc. All microarray data of this work is available from the NCBI-GEO website (http://www.ncbi.nlm.nih.gov/geo/) under GEO Series entry GSE79043.

\section{Modified Significance Analysis of Microarrays (SAM)}

The significance analysis of microarrays (http://statweb.stanford. edu/ tibs/SAM/) was used to identify the salinity induced, trait specific significant transcripts in diverse rice genotypes. The basic principle of SAM includes scoring each of the transcripts based on the changes in both gene expression and response variable. And it uses repeated permutations of the data to determine if the change in the expression of any gene is significantly related to the changes in response variable.

This study adopted a little modification as it analyzed the control and treated data separately. Unlike the unstressed condition, the stressed condition rendered substantial amount of variation in both gene expression and morpho-physiological trait responses in the eight diverse rice genotypes. So, it was only sensible to analyze the treated data separately (which otherwise is analyzed together in a mono-genotypic study) allowing SAM to identify the stress inducible significant transcripts.

For a particular trait (e.g., for shoot $\mathrm{Na}$ ) under stress condition, firstly the pre-processed (quantile normalized and $\log _{2}$ transformed) gene expression data of all the treated samples is arranged in a single spreadsheet (transcripts in rows; genotypes in column). The names of the samples (such as genotype-1, replication-1) in column heads are replaced by their corresponding trait responses which is weighted to a scale of 0 to 1 . The SAM program is then ran using the response type as "quantitative" based on 100 permutations using SAM version 4.0 software. The significant genes (relative to shoot $\mathrm{Na}$, in this instance) are selected based on the combined criteria of $\leq 5 \%$ FDR and $\geq 2$-fold score. To analyze the same for the rest of the traits, the sample names in the original spreadsheet is replaced by the weighted ranks $(0-1)$ of the trait in question. Further details of the SAM procedure used in this study can be found in Hossain (2014) and in SAM manual.

\section{Gene Ontology (GO) Enrichment Analysis}

The list of significant positively and negatively expressed genes were subjected to GO analysis using the AgriGO web-based tool (Du et al., 2010), available from http://bioinfo.cau.edu.cn/ agriGO/index.php. Singular Enrichment Analysis (SEA) (Huang et al., 2009) was used to identify the biological processes or 


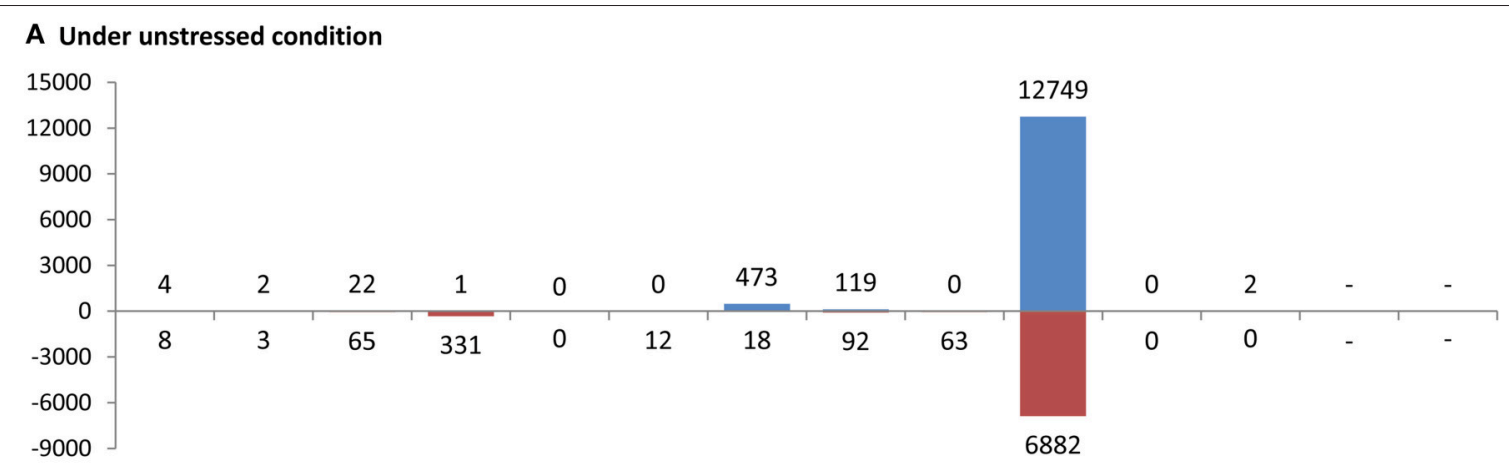

B Under stressed condition

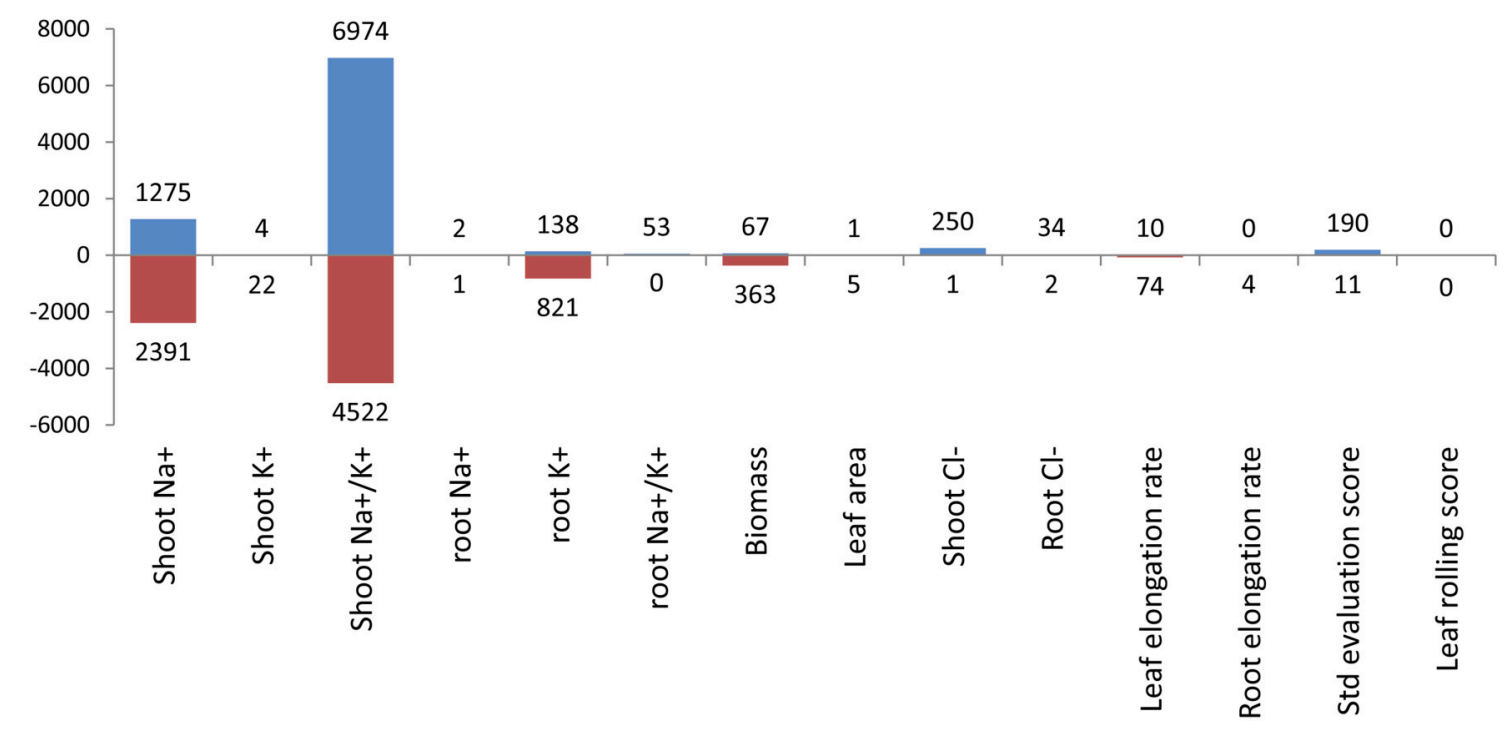

FIGURE 1 | Number of significant positively (blue bars) and negatively (red bars) expressed probes for each of the 14 morpho-physiological traits in the eight rice genotypes representing wide natural variation under unstressed $(A)$ and stressed (B) conditions as determined by the modified SAM approach. The complete lists of the significant probes along with the corresponding fold score (d), $q$-value and functional annotation can be found in Tables $\mathbf{S} 1 \mathbf{a}-\mathbf{n}$, S2a-n.

the molecular functions that are significantly enriched by the identified positively or negatively expressed genes for each of the traits. "Rice TIGR gene model" were used as reference during SEA.

\section{Mapping of the Genes on Chromosomes and the Regulatory Network of Genes}

The list of probes was mapped in the 12 chromosomes of rice using the Chromosome Map Tool (http://viewer.shigen.info/ oryzavw/maptool/MapTool.do) of GRAMENE genome browser database. The interactions between all the significant probes, as determined by SEA, were determined using the "The Rice Interactions Viewer" web based tool version Interactome 2.0 developed by the Bio-Analytic Resource- the BAR that queries a database of 37472 predicted and 430 confirmed Rice interacting proteins (http://bar.utoronto.ca/welcome.htm). Prior to using the "Rice Interactions Viewer," the RAP-DB gene Ids (e.g., Os06g0699400) were converted into MSU (TIGR) ID (e.g.,
LOC_Os06g48590) using the RiceXPro: Global gene expression profile web based tool (as shown in Table S4d).

\section{RESULTS AND DISCUSSION}

The gene expression data of eight rice genotypes were interrogated with their morpho-physiological data using modified SAM approach to identify the trait specific gene expression pattern in the wide genotypic background of rice under both stressed and unstressed conditions for each of the 14 morpho-physiological traits. The detailed morpho-physiological data (not shown here) are published as a separate article (Hossain et al., 2015a).

\section{Globally, More Genes are Expressed under Salt Stress}

In general, higher numbers of probes were found to be significantly expressed, both positively and negatively for most 
of the important salinity tolerance related traits $v i z$., shoot $\mathrm{Na}^{+}$, shoot $\mathrm{Na}^{+} / \mathrm{K}^{+}$, Biomass, root $\mathrm{K}^{+}$, and shoot $\mathrm{Cl}^{-}$under salt stress compared to that of unstressed conditions (Figure 1; Tables S1, S2). For example, 1275 and 2391 probes were positively and negatively expressed, respectively for shoot $\mathrm{Na}^{+}$, the single most important parameter to study salinity tolerance, under stressed condition compared to only 4 and 8 probes being positively and negatively expressed, respectively under unstressed conditions. Interestingly a very high number of genes were negatively expressed under stressed conditions, compared to that of under unstressed condition, for the trait "biomass" (Figure 1; Tables S1g, S2g, S3). All these probably indicate that plants deploy their adaptive mechanism by differentially activating a large number of genes under stressed conditions which otherwise is not activated constitutively.

Nonetheless, it is to keep in mind that these results are obtained by studying the RNA extracted from the whole seedling. And as gene expression, recently, is known to vary depending on cell or tissue types which can even be in opposite directions in some cases (Taylor-Teeples et al., 2011; Otsuki et al., 2014), verification of expression of all the identified genes in each cell or tissue types via in situ analysis will be helpful in revealing their exact roles in salinity tolerance mechanism.

\section{Genes Involved in Ion Homeostasis and Transport}

Lists of significant genes were mined manually to identify the putative genes that might be involved in ion transport with particular attention being given to the genes that were found to be significant for shoot $\mathrm{Na}$ and shoot $\mathrm{Na} / \mathrm{K}$. In total, 60 genes were found to be involved in ion homeostasis and transport processes (Table 2). Among the genes involved in sodium homeostasis, the notables are $\mathrm{Na}^{+} / \mathrm{H}^{+}$exchangers such as Os09g0286400, Os05g0382200, Os11g0648000, and Os12g0641100 and potassium transport related genes such as Os03g0656500, Os07g0102100, Os03g0337500, Os01g0932500, Os02g0519100, Os03g0575200, and Os04g0682800 etc. Genes for other cations such as $\mathrm{Ca}^{2+}, \mathrm{Mg}^{2+}$ and anions such as ammonium, nitrate, sulfate, and phosphate were also significantly expressed under salt stress.

Several membrane intrinsic and ion channel related genes having putative roles in ion homeostasis were also found to be significantly expressed e.g., aquaporin (Os09g0541000); membrane transporter (Os01g0704100), tonoplast integral protein (Os01g0975900, Os05g0231700); vesicle associated membrane protein (Os12g0639800), and ion channels (Os04g0643600, Os06g0527400, Os02g0255000, Os03g0758300, Os02g0117500, and Os01g0588200) etc. The cation transporter family protein (Os06g0701600) and cation/proton exchanger (Os01g0557500) are found to be negatively expressed for shoot Na.

Around 15 genes such as Os01g0290800, Os01g0356000, Os01g0609200, Os01g0609300, Os01g0966100 etc., were found to be involved in $\mathrm{ABC}$ transport (not shown in table). The ABCtransporter proteins are believed to transport various substrates such as ions, amino acids, sugars and peptides across cellular membranes besides their role in detoxification, plant growth and developmental processes (Martinoia et al., 2002; Davidson et al., 2008). In yeast, $A B C$ transporters are found to be involved in cation homeostasis but their role in plants is yet to be identified (Rea, 1999; Kang et al., 2010).

\section{Global Regulation of Biological Processes (BP) under Salt Stress}

More biological processes (BP) are significantly enriched by the induced genes (Table 3; Tables S4b,c) compared to that of by the constitutive genes (Tables S5b,c) as determined by the Singular Enrichment Analysis (SEA). Under unstressed conditions, no BPs were significantly enriched by the genes that are expressed for shoot $\mathrm{Na}^{+}$and shoot $\mathrm{Na}^{+} / \mathrm{K}^{+}$(Table S5b1), whereas most of the BPs were enriched by the genes that are expressed for these tissue ion traits under stressed conditions (Table 3; Table S4b1). This clearly shows that salt stress activates a series of genes which enrich different BPs in response to the stress across the range of genotypes. The major BPs that are activated under stressed conditions are Apoptosis, Stress Response, Signaling process, Transport, Metabolic and Catabolic process, Cellular, and Developmental processes etc (Table 3). The role of individual genes is not described in detail in this section, instead is discussed according to the molecular functions enriched by these genes in the next section.

\section{Global Regulation of Molecular Functions (MF) under Salt Stress}

Salt stress significantly enriches more molecular functions (MF) in the wide genetic background of rice compared to unstressed conditions as determined by the SEA (Table 4; Tables S4a,c, S5a,c). Significant positively and negatively expressed transcripts under salt stress enriched a number of MFs that includes signal transducer activity, transcription, and translation factory activity, serine hydrolase and metalloexopeptidase activity etc., (Table 4). The individual genes of important molecular functions are discussed below and the detailed discussion of all MFs can be found in Hossain (2014).

\section{Signal Transducer Activity}

Stress is first sensed by the receptors in membranes, which then generates secondary signal messengers like calcium, reactive oxygen species, kinases, and phosphates followed by the activation of transcription factor genes that eventually coordinates the plant's adaptive biochemical and physiological responses (Huang et al., 2012; Proietti et al., 2013). This study indentified 107 up-regulated and 54 down-regulated transcripts that are involved in signaling (Table 5).

Transcripts of three receptor like kinases (RLK) such as Os08g0442700, Os07g0134200, and Os06g0334300 are upregulated. RLKs are known to play roles in stress defense by sensing the extracellular signals and activating downstream pathways by phosphorylating target proteins (Marshall et al., 2012; Tanaka et al., 2012). Very recently, in rice, a putative RLK gene, OsSIK1, with extracellular leucine-rich repeats (Ouyang et al., 2010) and a cysteine-rich repeat (CRR) RLK sub-family 
TABLE 2 | Lists of genes (among the significant genes for (a) shoot $\mathrm{Na}^{+}$and (b) shoot $\mathrm{Na}^{+} / \mathrm{K}^{+}$) involved in ion homeostasis and transports under salinity stress in wide natural variation of rice genotypes.

\begin{tabular}{|c|c|c|c|c|c|}
\hline \multirow[t]{2}{*}{ Probe Name } & \multirow[t]{2}{*}{ Annotation } & \multicolumn{2}{|c|}{$\begin{array}{l}\text { (a) among the significant genes } \\
\text { for shoot } \mathrm{Na}^{+}\end{array}$} & \multicolumn{2}{|c|}{$\begin{array}{c}\text { (b) among the significant genes } \\
\text { for shoot } \mathrm{Na}^{+} / \mathrm{K}^{+}\end{array}$} \\
\hline & & Fold score & $q$-value (\%) & Fold score & $q$-value (\%) \\
\hline Os01g0557500 & Cation/proton exchanger $1 \mathrm{a}$. & -2.48 & 3.03 & & \\
\hline Os01g0645200 & Bile acid:sodium symporter family protein. & -2.35 & 3.35 & & \\
\hline Os05g0382200 & $\mathrm{Na}+/ \mathrm{H}+$ exchangeing protein-like. & & & 2.02 & 3.29 \\
\hline Os08g0503700 & Sodium/sulfate symporter family protein. & & & -2.26 & 2.81 \\
\hline Os09g0286400 & Sodium/hydrogen exchanger family protein. & & & 2.25 & 2.34 \\
\hline Os09g0299400 & Sodium-and chloride-activated ATP-sensitive potassium channel. & & & -2.72 & 1.79 \\
\hline Os09g0484900 & Sodium-dicarboxylate cotransporter-like. & & & -2.01 & 3.89 \\
\hline Os10g0436900 & Sodium/calcium exchanger membrane region domain cont. protein. & -2.13 & 4.88 & & \\
\hline Os01g0210700 & Potassium channel (Fragment). & -2.08 & 4.88 & & \\
\hline Os01g0369300 & Potassium transporter 1 (AtPOT1) (AtKUP1) (AtKT1). & -2.11 & 4.88 & & \\
\hline Os01g0648000 & Potassium channel. & & & 2.64 & 1.36 \\
\hline Os01g0696100 & K+ channel, two pore family protein. & & & 2.33 & 1.79 \\
\hline Os01g0932500 & K+ potassium transporter family protein. & & & 2.65 & 1.36 \\
\hline Os02g0519100 & K+ potassium transporter family protein. & & & 2.40 & 1.79 \\
\hline Os02g0612700 & $\mathrm{K}+$ channel tetramerisation domain containing protein. & -2.52 & 2.51 & & \\
\hline Os03g0337500 & K+ potassium transporter family protein. & -3.57 & 0.71 & & \\
\hline Os03g0575200 & K+ potassium transporter family protein. & & & 1.88 & 3.89 \\
\hline Os03g0656500 & K-exchanger-like protein. & -2.24 & 3.62 & & \\
\hline Os01g0908500 & Mg2+ transporter protein, CorA-like family protein. & -2.13 & 4.88 & & \\
\hline Os02g0138900 & Low affinity calcium antiporter CAX2. & -2.54 & 2.51 & & \\
\hline Os02g0720700 & $\mathrm{Cl}$ - channel, voltage gated family protein. & & & 1.99 & 3.29 \\
\hline Os04g0605500 & Calcium-transporting ATPase 8, plasma membrane-type & -2.93 & 1.44 & & \\
\hline Os04g0653200 & Low affinity calcium transporter CAX2 (Fragment). & & & 1.88 & 3.89 \\
\hline Os05g0594200 & Calcium/proton exchanger superfamily protein. & 2.65 & 3.03 & & \\
\hline Os03g0150800 & High affinity phosphate transporter 2 (Phosphate transporter). & & & 2.91 & 0.88 \\
\hline Os03g0161200 & Sulfate transporter 3.1 (AST12) (AtST1). & -2.95 & 1.30 & & \\
\hline Os03g0195800 & High affinity sulfate transporter. & & & 3.62 & 0.38 \\
\hline Os03g0838400 & Ammonium transporter. & -2.99 & 1.30 & & \\
\hline Os04g0185600 & Phosphate transporter 6. & -3.65 & 0.71 & & \\
\hline Os05g0477800 & High-affinity sulfate transporter HvST1. & & & 2.69 & 1.09 \\
\hline Os08g0155400 & Nitrate transporter (Fragment). & -2.67 & 2.15 & & \\
\hline Os08g0406400 & Sulfate transporter (Fragment). & 2.67 & 2.51 & & \\
\hline Os09g0240500 & Sulfate transporter 4.1, chloroplast precursor (AST82). & -2.26 & 3.62 & & \\
\hline Os10g0444600 & Phosphate transporter (Fragment). & -2.50 & 2.51 & & \\
\hline Os01g0588200 & Voltage-dependent anion channel. & & & 2.78 & 1.09 \\
\hline
\end{tabular}


TABLE 2 | Continued

\begin{tabular}{|c|c|c|c|c|c|}
\hline \multirow[t]{2}{*}{ Probe Name } & \multirow[t]{2}{*}{ Annotation } & \multicolumn{2}{|c|}{$\begin{array}{l}\text { (a) among the significant genes } \\
\text { for shoot } \mathrm{Na}^{+}\end{array}$} & \multicolumn{2}{|c|}{$\begin{array}{c}\text { (b) among the significant genes } \\
\text { for shoot } \mathrm{Na}^{+} / \mathrm{K}^{+}\end{array}$} \\
\hline & & Fold score & $q$-value (\%) & Fold score & $q$-value (\%) \\
\hline Os01g0704100 & Membrane transporter. & -2.52 & 2.51 & & \\
\hline Os01g0975900 & Tonoplast membrane integral protein ZmTIP1-2. & 3.31 & 0.80 & & \\
\hline Os02g0117500 & Glutamate receptor 3.2 precursor (AtGluR2). & & & 3.38 & 0.39 \\
\hline Os02g0255000 & Cyclic nucleotide-gated ion channel 1 (AtCNGC1) & & & 2.09 & 2.81 \\
\hline Os02g0823100 & Plasma membrane intrinsic protein (ZmPIP1-5) & -2.61 & 2.15 & & \\
\hline Os03g0129100 & Seven transmembrane protein MLO2. & -2.78 & 1.92 & & \\
\hline Os03g0758300 & Cyclic nucleotide-gated ion channel 2 (AtCNGC2) & -2.35 & 3.35 & & \\
\hline Os04g0643600 & Cyclic nucleotide-gated channel C (Fragment). & -2.13 & 4.88 & & \\
\hline Os05g0231700 & Tonoplast membrane integral protein ZmTIP4-2. & 3.35 & 0.67 & & \\
\hline Os06g0527400 & Cyclic nucleotide-gated calmodulin-binding ion channel. & & & 1.84 & 3.89 \\
\hline Os08g0555000 & Transmembrane 9 superfamily protein member 2 precursor (p76). & 2.83 & 1.92 & & \\
\hline Os09g0541000 & Plasma membrane intrinsic protein 2c, (PIP2c, TMP2C) & 2.56 & 3.35 & & \\
\hline Os12g0639800 & Vesicle-associated membrane protein 722 (AtVAMP722) & -2.70 & 1.92 & & \\
\hline
\end{tabular}

gene, ARCK1 (Tanaka et al., 2012) was reported to be induced by salt and drought stresses.

Transcripts of 5 Ethylene receptor genes such as Os05g0155200, Os07g0259100, Os03g0701700, Os04g0169100, and Os02g0820900 were found to be positively expressed under salt stress. The role of ethylene in salt stress response is reported to be equivocal (Achard et al., 2006) and In tobacco, an ethylene receptor gene, NTHK1 was reported to promote leaf growth (Cao et al., 2006), which demonstrated the significance of these ethylene receptor genes to be studied further in determining their role in salt tolerance.

Among the leucine-rich repeat containing transcripts, three (Os01g0239700, Os08g0446400, and Os01g0140400) and two (Os06g0717200 and Os11g0514500) were found to be negatively regulated in this study, respectively. Cheng et al. (2009) reported a leucine-rich-repeat type receptor-like protein kinase, OsRPK1 to be induced by multiple stresses in plasma membrane of cortex cells in rice roots and Lorenzo et al. (2009) reported an increase in expression of leucine-rich gene, Srlk in Medicago truncatula roots upon exposure to salt stress. The Srlk gene also had a homolog, Os05g0414700, which was also found to be positively expressed in this study.

Protein kinases regulate the phosphorylation and dephosphorylation of other proteins and play a crucial role in stress signal transduction. To date, several plant protein kinases, particularly the mitogen-activated protein (MAPK/MPK) kinase are reported to be activated by osmotic stress (Fujita et al., 2006; Sinha et al., 2011). Five MAP kinases including MAP kinase 2 (Os06g0699400), 6 (Os06g0154500) and MAPK homolog MMK2 (Os10g0533600) were positively regulated and three MAP kinases (Os06g0708000, Os06g0367900, and Os05g0566400) are negatively expressed in wide natural rice variation (Table 5). In addition, Serine/threonine protein kinases have also been known to be involved in multi-stress tolerance in plants (Zhao et al., 2009). Among the serine/threonine kinases, four (Os01g0323000, Os01g0631700, Os10g0136400, and Os07g0537200) was positively expressed and only one (Os06g0496800) was found to be negatively regulated. At least, 33 and 13 protein kinase domain containing proteins were found to be positively and negatively expressed in the wide natural variation of rice genotypes.

Among the many other positively expressed transcripts, notable are heat shock protein, mannose binding, extensins, histidin kinases, NPH3 domain containing protein, and Znfinger domain containing proteins. These transcripts were found to be located in all the chromosomes except in chromosome 11 and chromosomes 1, 2, 6, and 7 contained most of the genes (Figure S1).

\section{Transcription Factor (TF) Activity}

The rice genome is known to have 1772 TFs that generally falls under the family of WRKY, DREB, CBF, MYB, bZIP, ERF, zinc-finger, helix-loop-helix, and NAC (Sreenivasulu and Miranda, 2004; Duque et al., 2013). Of the negatively expressed transcripts for shoot $\mathrm{Na}^{+} / \mathrm{K}^{+}, 81$ transcripts were found to significantly enrich the "Transcription factor activity" (Table 6 and Table S4a2). The four bZip family TFs (Os01g0542700 encoding OsbZIP4, Os03g0770000 encoding OsbZIP32, Os08g0543900 encoding OsbZIP68, and Os11g0154800) that were found to be salt responsive in this study have not been reported before. Some of the identified salt and drought responsive bZIP proteins are $O z B Z 8$ (Mukherjee et al., 2006), OsbZIP15 (Zou et al., 2008), OsbZIP23 (Xiang et al., 2008), OsbZIP46 (Tang et al., 2012), OzAREB1 (Jin et al., 2010), and OsbZIP16 (Chen et al., 2012).

Among the 70 identified WRKY genes in rice and Arabidopsis (Goff et al., 2002; Dong et al., 2003), transcripts of 12 TFs were found to be salt stress responsive in this study (Table 6 and Table S4a2). Of these, OsWRKY6 (Os03g0798500) and OsWRKY42 (Os02g0462800) were found to be low phosphorus (Chen et al., 2009) and low boron (Kasajima et al., 2010) responsive, OsWRKY24 (Os01g0826400) stress responsive (Wei 
TABLE 3 | List of significant GO categories of biological process under stressed condition for significant positively and negatively expressed genes for different morpho-physiological traits as determined by Singular Enrichment Analysis.

\section{GO Category: Biological Processes}

Programmed cell death

Apoptosis

Response to abiotic stimulus

Stress Response

Response to abiotic stimulus
Response to chemical stimulus (response to
endogenous, organic substance, and hormone)
Response to biotic stimulus
Cellular response to stimulus

Cellular response to stimulus

8

Signal transduction, intracellular signaling process; signaling pathway

Signaling process

Transport

Transmembrane transport

Di-, tri-valent inorganic cation transport; and transition metal ion transport

Metabolic processes

Regulation of transcription, gene expression

Positively Expressed Genes

Negatively Expressed Genes

Shoot $\mathrm{Na}^{+}$Shoot $\mathrm{Na}^{+} / \mathrm{K}^{+}$Root $\mathrm{K}^{+}$Shoot $\mathrm{Na}^{+}$Shoot $\mathrm{Na}^{+} / \mathrm{K}^{+} \operatorname{Root} \mathrm{K}^{+}$

9

\begin{tabular}{lllll} 
& 29 & 12 & 29 & \\
\hline & 110 & 19 & 77 & 6 \\
\hline & 14 & & 11 & \\
\hline & 77 & & 46
\end{tabular}

Negative regulation of gene expression (silencing)

177

Translation

45

Cellular nitrogen compound biosynthetic process (amine, amino acid biosynthetic process)

\begin{tabular}{|c|c|c|c|c|c|c|}
\hline $\begin{array}{l}\text { Protein modification by small protein conjugation or } \\
\text { removal }\end{array}$ & & 31 & & 19 & 22 & \\
\hline Protein amino acid dephosphorylation & & & & 16 & & \\
\hline $\begin{array}{l}\text { Generation of precursor metabolites and energy } \\
\text { (including photosynthesis, light harvesting) }\end{array}$ & & 26 & & 43 & 67 & \\
\hline Small molecule metabolic process & & 15 & & & 208 & \\
\hline Cellular lipid metabolic process & & & & & 51 & \\
\hline Cellulose metabolic process & & 17 & & & & \\
\hline Secondary metabolic process & & 31 & & 11 & 34 & \\
\hline Including protein, polysaccharadie catabolic process & & 126 & & & & \\
\hline Cell cycle & & 17 & & 7 & 23 & \\
\hline DNA conformation change (DNA packaging) & 16 & & & & & \\
\hline DNA recombination & & 32 & & & & \\
\hline Microtubule cytoskeleton organization & & 6 & & & & \\
\hline Cellular macromolecular complex subunit organization & 19 & & & & & \\
\hline Multicellular organismal process & 8 & 88 & & 27 & 31 & 10 \\
\hline Cellular cell wall organization or biogenesis & & 70 & & 9 & 13 & \\
\hline Reproduction & & 66 & & 18 & & \\
\hline Regulation of anatomical structure size & & 9 & & & & \\
\hline Oxidation reduction & 29 & & 6 & 54 & & 12 \\
\hline
\end{tabular}

The details (GO terms, p-values, FDR values, GO flash charts and schematic diagrams) can be found in Tables S4b1,2.

9

61

$9 \quad 13$

9

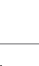


TABLE 4 | List of significant GO categories of molecular function under stressed condition for significant positively and negatively expressed genes for different morpho-physiological traits as determined by Singular Enrichment Analysis.

\begin{tabular}{|c|c|c|c|c|c|c|c|c|}
\hline \multicolumn{2}{|c|}{ GO category: Molecular Functions } & \multicolumn{3}{|c|}{ Positively expressed genes } & \multicolumn{4}{|c|}{ Negatively expressed genes } \\
\hline & & Shoot $\mathrm{Na}^{+}$ & Shoot $\mathrm{Na}^{+} / \mathrm{K}^{+}$ & Root $\mathrm{K}^{+}$ & Shoot $\mathrm{Na}^{-}$ & Shoot $\mathrm{Na}^{+} / \mathrm{K}^{+}$ & Root $\mathbf{K}^{+}$ & Biomass \\
\hline \multicolumn{2}{|c|}{ Signal transducer activity } & & 107 & & & 56 & & \\
\hline \multirow{7}{*}{ 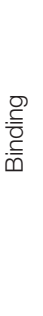 } & Transcription factor activity & & & & 81 & & & \\
\hline & Translation factor activity (nucleic acid binding) & & 36 & & & 26 & & \\
\hline & SNAP receptor activity & & & & & 6 & & \\
\hline & Chaperone binding & & & & & 6 & & \\
\hline & Manganese ion binding & & & & 18 & 18 & & \\
\hline & $\begin{array}{l}\text { Alkali metal ion binding (including potassium ion } \\
\text { binding) }\end{array}$ & & 8 & & & & & \\
\hline & 2 iron, 2 sulfur cluster binding & & & & & 5 & & \\
\hline \multirow{7}{*}{ 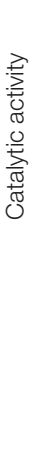 } & $\begin{array}{l}\text { Phosphoprotein phosphatase activity (including } \\
\text { protein serine/threonine phosphatase activity) }\end{array}$ & & & & 21 & & & \\
\hline & Protein methyltransferase activity & & 13 & & & & & \\
\hline & $\begin{array}{l}\text { Serine O-acyltransferase activity (including serine } \\
\text { O-acetyltransferase activity) }\end{array}$ & & & & 5 & & & \\
\hline & $\begin{array}{l}\text { Serine hydrolase activity (including endopeptidase } \\
\text { activity) }\end{array}$ & 10 & 55 & & & 25 & & 5 \\
\hline & Metalloexopeptidase activity & & 16 & & & 9 & & \\
\hline & $\begin{array}{l}\text { Oxidoreductase activity, acting on } \mathrm{CH}-\mathrm{OH} \text { group of } \\
\text { donors }\end{array}$ & & & & & 39 & & \\
\hline & $\begin{array}{l}\text { Oxidoreductase activity, acting on single donors } \\
\text { with incorporation of molecular oxygen }\end{array}$ & & & & & 12 & & \\
\hline \multicolumn{2}{|c|}{ Electron carrier activity } & 27 & 125 & 6 & 48 & 109 & 17 & \\
\hline
\end{tabular}

The details of the GO terms, p-values, FDR values, GO flash charts and schematic diagrams can be found in Tables S4a1,a2,c.

et al., 2013); OsWRKY34 (Os01g0665500 and Os07g0583700) cold responsive (Zou et al., 2010); OsWRKY34 (Os02g0265200) heat responsive; all in Arabidopsis (Li et al., 2010). However, the TFs WRKY31 (Os01g0750100), WRKY32 (Os02g0770500), WRKY44 (Os08g0276200), WRKY49 (Os01g0730700), WRKY63 (Os06g0158100), and WRKY71 (Os02g0181300) were not reported earlier.

The other important TFs found to be salt responsive in this study include CBF like protein, E2F protein, ethylene responsive TFs, heat shock, and MADS-box proteins and AP2 domain containing proteins (Table 6 and Table S4a2). Chromosome 1, 2 , and 3 contain most of the genes while no genes were located in chromosome 10 and 12 (Figure S2). Some of these TFs are reported to play roles in abiotic and biotic stress tolerance in rice and other crops; however, a detailed investigation of the previously unidentified TFs will provide valuable information in explaining salinity tolerance mechanisms in rice.

\section{Translation Factor Activity}

The regulation of translation, that facilitates the selective synthesis of required proteins, is one of the versatile strategies plants have evolved to cope with the environmental stresses. Generally, in eukaryotes, eukaryotic initiation factor (eIF4E) recognizes the $5^{\prime}$-cap structure of mRNAs to begin the canonical cap-dependent translation. The eIF4G and $e I F 4 A$ then interacts with eIF4E to form the eIF4F (cap-binding complex) and the factors $e I F 4 B, e I F 3, e I F 1$, and $e I F 1 A$ are subsequently recruited that ultimately regulates translation (Echevarría-Zomeño et al., 2013). Under stresses, the eukaryotic cells usually inhibit this translation initiation that affects the activity of initiation factor eIF2 and eIF4E, a mechanism mainly unknown in plants (Wek et al., 2006; Muñoz and Castellano, 2012). In this study, among the significantly positively and negatively expressed genes, 36 and 26 genes, respectively were found to enrich the translation factor activity (Table 7, Table S4a2; Figure S3).

Among the up-regulated genes the most noticeable fall under the category of translation initiation factors such as Os02g0146600 (eIF4A), Os03g0566800 (eIF4A-3), Os05g0566500 (eIF-3 zeta), Os07g0124500 (eIF3 p110), Os07g0555200 (eIF4G), Os07g0597000 (eIF-5A), Os03g0758800, and Os12g0507200 (eIF-5A) (eIF-4D), Os05g0592600 (eIF 2 family protein), Os02g0101100 (eIF 3 family protein) and Os02g0557600, and Os05g0498400 (IF SUI1 family protein); transcription elongation factors such as Os03g0196900 (TFIIB), Os11g0166800 (TFS-II), Os03g0441000 and Os01g0846900 (TFIID), Os07g0662500 (EF-1-beta'), Os06g0571400 (EF-1-gamma), Os01g0742200 (EF-2), and Os03g0565500(mEF-G-1) etc.

Several elongation factors e.g., Os03g0177400 and Os03g0178000 (EF-1 $\alpha$ ), Os11g0116400 (EF-P), Os12g0541500 
TABLE 5 | Lists of positively (a) and negatively (b) expressed significant transcripts (for shoot $\mathrm{Na}^{+} / \mathrm{K}^{+}$) that are involved in the Molecular Function "Signal transducer activity" as determined by the Singular Enrichment Analysis (SEA).

\section{Name}

(a) Positively expressed transcripts (107)

Os08g0442700, Os07g0134200, Os06g0334300

Os07g0107800

Os05g0155200, Os07g0259100, Os03g0701700, Os04g0169100, Os02g0820900

Os07g0132500

Os01g0239700, Os08g0446400, Os01g0140400

Os01g0836800

Os07g0522600

Os02g0131600

Os02g0117500

Os02g0245100

Os06g0225300

Os01g0665200

Os06g0699400

Os05g0576800

Os06g0154500

Os10g0533600

Os09g0349800, Os09g0349600, Os08g0493800, Os04g0540900, Os02g0111800, Os06g0693200, Os02g0153200, Os04g0658700, Os03g0791700, Os05g0525600, Os08g0203700, Os02g0151100, Os01g0976900, Os10g0155800, Os01g0960400, Os10g0497600, Os01g0664200, Os01g0110500, Os01g0741200, Os02g0218400, Os02g0227700, Os02g0153100, Os03g0148700, Os06g0693000, Os01g0514700, Os01g0114900, Os01g0738300 Os05g0414700, Os06g0654600

Os10g0533800, Os07g0131100, Os03g0772600, Os12g0562500

Os01g0323000, Os01g0631700, Os10g0136400

Os07g0537200

Os01g0223900, Os02g0527900

Os02g0150800

Os12g0256000, Os05g0407500

Os07g0613300

Os03g0284100

Os03g0637600

Os08g0332800

Os08g0230300

Os06g0199800

Os06g0111400

Os06g0163000

Os01g0923700

Os01g0114700

Os07g0584200, Os04g0477000

Os06g0625300

Os06g0687800

Os07g0130700

Os08g0376700, Os02g0618200, Os06g0654300, Os09g0532400, Os03g0224200

Os07g0537900

Os05g0525000

\section{Annotation}

Receptor-like kinase, Receptor-like protein kinase 3

Phytosulfokine receptor precursor (EC 2.7.1.37)

Ethylene receptor, Ethylene receptor homolog, Ethylene receptor-like protein 2

Lectin-like receptor kinase 7;2

Leucine-rich receptor-like protein kinase

Lung seven transmembrane receptor family protein

Metabotropic $\gamma$-aminobutyric acid receptor, type B family protein

Mitochondrial import receptor subunit TOM22, TOM9 homolog

Glutamate receptor 3.2 precursor (AtGluR2)

Peroxisomal targeting signal type 2 receptor

Brassinosteroid insensitive 1-associated receptor kinase 1 precursor

Mitogen-activated protein kinase, Blast and wounding induced

MAP kinase 2

MAP kinase homolog

Mitogen-activated protein kinase (MAP kinase 6)

Mitogen-activated protein kinase homolog MMK2 (EC 2.7.1.37)

Protein kinase domain containing protein

Protein kinase family protein

Serine/threonine kinase, Ser Thr specific protein kinase-like protein

Curculin-like (mannose-binding) lectin domain containing protein

Cyclin-like F-box domain containing protein

Esterase/lipase/thioesterase domain containing protein

Exportin-t

Expressed protein (Pseudo-response regulator 9) (Timing of CAB expression 1-like protein) Extensin protein-like

F7018.23 protein (SWP1) (Struwwelpeter 1 protein)

Galactose oxidase, central domain containing protein

GPCR, family 2, secretin-like protein

Guanine nucleotide binding protein, alpha subunit family protein

Heat shock protein STI (Stress inducible protein) (GmSTI)

Histidine kinase-like protein

LRK33

NPH3 domain containing protein

Peptidoglycan-binding LysM domain containing protein

Pincher

Resistance protein candidate (Fragment)

Response regulator 1 , Response regulator receiver domain containing protein

SRK3 gene

TMK protein precursor 
TABLE 5 | Continued

\section{Name}

Os01g0904700, Os06g0183100

Os06g0574200, Os02g0218600

Os09g0416700

Os02g0205400

Os02g0830200, Os04g0524300

Os05g0112000, Os01g0974400, Os03g0275300

Os06g0716000, Os04g0433600

Os09g0573200, Os09g0470900

Os03g0738800, Os07g0501800, Os04g0631900,

Os01g0690900

\section{Annotation}

Two-component response regulator ARR1. Splice isoform 2

UspA domain containing protein

Vesicle transport $v$-SNARE family protein

WD40-like domain containing protein

ZmRR2 protein (Response regulator 2)

Zn-finger, RING domain containing protein

Protein of unknown function DUF668 family protein

Conserved hypothetical protein

Hypothetical protein

\section{(b) Negatively expressed transcripts (54)}

\section{Os10g0346600}

Os11g0473000

Os05g0529300

Os06g0680500

Os06g0717200

Os11g0514500

Os03g0343400

Os01g0176400

Os01g0114600

Os06g0496800

Os08g0480100

Os05g0100700, Os08g0174700

Os06g0708000

Os06g0367900

Os05g0566400

Os01g0206800, Os08g0203400, Os05g0588300,

Os05g0258400, Os05g0480400, Os02g0228300,

Os01g0116400, Os06g0676600, Os02g0821400,

Os01g0779300, Os02g0106900

Os11g0678000, Os01g0114100

Os01g0121100

Os01g0958100

Os03g0284100

Os03g0637600

Os07g0535700

Os06g0111400

Os11g0206700

Os05g0186100

Os01g0855600

Os02g0259100

Os02g0459600

Os11g0102200

Os03g0206700, Os12g0117600, Os11g0118300,

Os03g0347700, Os11g0118500

Os05g0127200

Os07g0694000

Os07g0695100

Os02g0729400

Os12g0117400

Os11g0143300

Os01g0560200, Os01g0707300
BP-80 vacuolar sorting receptor

ER lumen protein retaining receptor (HDEL receptor)

ER lumen protein retaining receptor (HDEL receptor)

Glutamate receptor 3.1 precursor (Ligand-gated ion channel) (AtGLR2)

Leucine-rich repeat/receptor protein kinase precursor

Leucine-rich repeat-containing extracellular glycoprotein precursor

Photolyase/blue-light pthotoreceptor (PHR2)

Photoreceptor-interacting protein-like

Receptor-like kinase ARK1AS (Fragment)

Serine/threonine kinase receptor precursor

Signal recognition particle receptor protein (Fragment)

Somatic embryogenesis receptor kinase-like protein

MAP kinase homolog

Mitogen-activated protein kinase homolog

Mitogen-activated protein kinase. Blast and wounding induced

Protein kinase domain containing protein

Protein kinase family protein

AR401

Cell division transporter substrate-binding protein, FtsY family protein

Expressed protein (Pseudo-response regulator 9) (Timing of CAB expression 1-like protein)

Extensin protein-like

F-box protein interaction domain containing protein

Guanine nucleotide binding protein (G-protein), alpha subunit family protein

Guanine nucleotide binding protein (G-protein)

Histidine-containing phosphotransfer protein 4

Hs1pro-1 protein

Hypothetical protein

Legume lectin, beta domain containing protein

$\mathrm{NPH} 1-1$

$\mathrm{NPH} 3$ domain containing protein

Phosphoinositide-specific phospholipase C (PLC) family protein

Phosphoinositide-specific phospholipase C

Response regulator receiver domain containing protein

Rhodanese-like domain containing protein

RPT2-like protein

Type-A response regulator

Vesicle transport v-SNARE family protein

The chromosomal distribution of the positively expressed genes is shown in Figure S1. 
TABLE 6 | Lists of significant transcripts (for shoot $\mathrm{Na}^{+} / \mathrm{K}^{+}$) that are involved in transcription factor activity as determined by the Singular Enrichment Analysis (SEA).

\begin{tabular}{|c|c|}
\hline Name & Annotation \\
\hline Os01g0952800 & $\begin{array}{l}\text { Basic helix-loop-helix dimerisation region bHLH domain } \\
\text { containing protein }\end{array}$ \\
\hline $\begin{array}{l}\text { Os01g0542700, } \\
\text { Os03g0770000 }\end{array}$ & $\begin{array}{l}\text { Basic-leucine zipper (bZIP) transcription factor domain } \\
\text { containing protein }\end{array}$ \\
\hline Os08g0543900 & BZIP transcription factor RF2b \\
\hline Os11g0154800 & DNA-binding factor of bZIP class (Fragment) \\
\hline Os01g0826400 & WRKY transcription factor 24 \\
\hline Os01g0750100 & WRKY transcription factor 31 \\
\hline Os02g0770500 & WRKY transcription factor 32 \\
\hline $\begin{array}{l}\text { Os01g0665500, } \\
\text { Os07g0583700 }\end{array}$ & WRKY transcription factor 34 \\
\hline Os02g0265200 & WRKY transcription factor 39 \\
\hline Os02g0462800 & WRKY transcription factor 42 (Transcription factor WRKY02) \\
\hline Os08g0276200 & WRKY transcription factor 44 (WRKY DNA-binding protein 44) \\
\hline Os01g0730700 & WRKY transcription factor 49 \\
\hline Os03g0798500 & WRKY transcription factor 6 \\
\hline Os06g0158100 & WRKY transcription factor 63 \\
\hline Os02g0181300 & WRKY transcription factor 71 (Transcription factor WRKY09) \\
\hline Os03g0680800 & BEL1-related homeotic protein 14 (Fragment) \\
\hline Os03g0762000 & Casein kinase II alpha subunit \\
\hline Os06g0127100 & CBF-like protein \\
\hline Os02g0203000 & $\begin{array}{l}\text { Concanavalin A-like lectin/glucanase domain containing } \\
\text { protein }\end{array}$ \\
\hline Os04g0597300 & DNA-binding WRKY domain containing protein \\
\hline Os01g0678700 & DP protein \\
\hline Os01g0165000 & DRE binding protein 2 \\
\hline Os03g0152100 & E2F dimerization factor \\
\hline Os02g0537500 & E2F homolog \\
\hline Os04g0669200 & Ethylene response factor 3 \\
\hline Os05g0497200 & Ethylene responsive element binding factor 4 (AtERF4) \\
\hline $\begin{array}{l}\text { Os05g0497300, } \\
\text { Os06g0194000 }\end{array}$ & Ethylene responsive element binding factor 5 (AtERF5) \\
\hline Os02g0655200 & Ethylene responsive element binding factor3 (OsERF3) \\
\hline Os01g0934300 & Flowering-time protein isoform beta \\
\hline Os01g0658900 & G-box binding factor 1 \\
\hline Os03g0640800 & HD-Zip protein (Homeodomain transcription factor) (ATHB-14) \\
\hline Os03g0745000 & $\begin{array}{l}\text { Heat shock factor (HSF)-type, DNA-binding domain } \\
\text { containing protein }\end{array}$ \\
\hline Os02g0232000 & Heat shock transcription factor 29 (Fragment) \\
\hline Os03g0854500 & Heat shock transcription factor 31 (Fragment) \\
\hline Os06g0603000 & Heme oxygenase 1 (Fragment) \\
\hline Os02g0147800 & Homeo protein (Fragment) \\
\hline $\begin{array}{l}\text { Os01g0818400, } \\
\text { Os03g0109400, } \\
\text { Os04g0541700, } \\
\text { Os04g0548700 }\end{array}$ & Homeobox domain containing protein \\
\hline Os05g0129700 & Homeobox protein rough sheath 1 \\
\hline Os03g0188900 & $\begin{array}{l}\text { Homeobox-leucine zipper protein ATHB-6 (Homeodomain } \\
\text { transcription factor ATHB-6) (HD-ZIP protein ATHB-6) }\end{array}$ \\
\hline Os09g0528200 & Homeodomain leucine zipper protein (Fragment) \\
\hline Os03g0224700 & HSP protein (Fragment) \\
\hline
\end{tabular}

(Continued)
TABLE 6 | Continued

\begin{tabular}{ll}
\hline Name & Annotation \\
\hline Os03g0673000 & Knotted1-type homeobox protein OSH10 (Fragment) \\
Os03g0727200 & Knotted1-type homeobox protein OSH3 \\
Os01g0201700 & MADS box protein \\
Os06g0712700 & MADS-box protein SPW1 \\
Os05g0437700 & OSE2-like protein (Fragment) \\
Os02g0797100, & Pathogenesis-related transcriptional factor and ERF domain \\
Os04g0547600, & containing protein \\
Os04g0610400, & \\
Os05g0549800, & \\
Os06g0691100, & \\
Os07g0227600, & \\
Os08g0408500, & \\
Os08g0521600, & \\
Os11g0129700 & \\
Os01g0883100 & PISTILLATA-like MADS box protein \\
Os01g0174000 & Protein HY5 (AtbZIP56) \\
Os06g0712600 & Protein of unknown function DUF702 family protein \\
Os02g0649300 & Short highly repeated, interspersed DNA (Fragment) \\
Os06g0252300 & TF-like protein (Fragment) \\
Os01g0899800, & ANT (Ovule development protein aintegumenta) \\
Os06g0657500 & \\
Os03g0341000 & AP2 domain containing protein RAP2.2 (Fragment) \\
Os03g0191900 & AP2 domain family transcription factor homolog (AP2 domain \\
Os09g0369000 & AP2 domain transcription factor RAP2.3 (Related to AP2 \\
Os02g0657000 & AP2 domain-containing protein Rap211 \\
Os09g0423800 & AP2-1 protein (Fragment) \\
Os02g0546600 & AP2-domain DNA-binding protein \\
Os04g050539500 & AtGATA-3 protein (GATA transcription factor 3) \\
Os03g0313100, & BABY BOOM \\
Os08g0442400 & \\
Os08g0472400, & Conserved hypothetical protein \\
Os031000 & Hypothetical protein \\
\hline
\end{tabular}

The chromosomal distribution of the positively expressed genes is shown in Figure $\mathbf{S 2}$.

(EF-Ts), Os07g0614500 (EF-1-beta), and Os02g0220500 and Os02g0220600 (EF-1-gamma) etc., and several translation initiation factors e.g., Os02g0300700 (EIF-1A), Os07g0681000 (eIF-2-beta) (P38), Os01g0120800 (eIF-3 theta), Os07g0167000 (eIF-3 p48), Os01g0970400 (eIF4E-1), Os02g0794400 (IF-3 family protein), Os05g0107700 (TFIIA-gamma), and Os07g0639800 (IF6 family protein) etc., were also found to be negatively expressed.

\section{SNAP Receptor and Chaperone Activity}

SNAP receptor activity is regulated by a super family of proteins known as SNAREs [soluble $\mathrm{N}$-ethylmaleimide-sensitive factor (NSF) attachment protein receptors] that act as a marker to identify a membrane and selectively interact with SNAREs on other membrane surfaces to mediate membrane fusion thus providing a continuous flux of membranes via transport vesicles. This vesicle traffic is believed to be involved in cell homeostasis, 
TABLE 7 | Lists of positively (a) and negatively (b) expressed transcripts (for shoot $\mathrm{Na}^{+} / \mathrm{K}^{+}$) that significantly enriched the translation factor activity in wide natural variation of rice genotypes.

\section{Name Annotation}

(a) Positively expressed transcripts (36)

\begin{tabular}{|c|c|}
\hline Os02g0146600 & Eukaryotic initiation factor $4 \mathrm{~A}$ (elF4A) (elF-4A) \\
\hline Os03g0566800 & Eukaryotic initiation factor 4A-3 (elF4A-3) (elF-4A-3) \\
\hline Os05g0566500 & $\begin{array}{l}\text { Eukaryotic translation initiation factor } 3 \text { subunit } 7 \text { (elF-3 zeta) } \\
\text { (elF3d) (p66) }\end{array}$ \\
\hline Os07g0124500 & $\begin{array}{l}\text { Eukaryotic translation initiation factor } 3 \text { subunit } 8 \text { (elF3 p110) } \\
\text { (elF3c) }\end{array}$ \\
\hline Os07g0555200 & Eukaryotic translation initiation factor $4 \mathrm{G}$ \\
\hline Os07g0597000 & Eukaryotic translation initiation factor $5 \mathrm{~A}$ (elF-5A) \\
\hline Os03g0758800 & Eukaryotic translation initiation factor $5 \mathrm{~A}-2$ (elF-5A) (elF-4D) \\
\hline Os12g0507200 & Eukaryotic translation initiation factor 5A-2 (elF-5A) (elF-4D) \\
\hline Os05g0592600 & Initiation factor 2 family protein \\
\hline Os02g0101100 & Initiation factor 3 family protein \\
\hline Os05g0575300 & $\begin{array}{l}\text { Translation initiation factor IF-2, chloroplast precursor } \\
\text { (PvIF2cp) }\end{array}$ \\
\hline $\begin{array}{l}\text { Os02g0557600, } \\
\text { Os05g0498400 }\end{array}$ & Translation initiation factor SUl1 family protein \\
\hline Os04g0237300 & DNA-directed RNA polymerase alpha chain (EC 2.7.7.6) (PEP) \\
\hline Os03g0851100 & Eftu \\
\hline Os03g0196900 & TFIIB-related protein (Fragment) \\
\hline Os11g0166800 & $\begin{array}{l}\text { Transcription elongation factor S-II, N-terminal domain } \\
\text { containing protein }\end{array}$ \\
\hline Os03g0441000 & $\begin{array}{l}\text { Transcription initiation factor TFIID component TAF4 domain } \\
\text { containing protein }\end{array}$ \\
\hline Os01g0846900 & Transcription initiation factor TFIID domain containing protein \\
\hline Os07g0662500 & Elongation factor 1-beta' (EF-1-beta') \\
\hline Os06g0571400 & Elongation factor 1-gamma (EF-1-gamma) (eEF-1B gamma) \\
\hline Os01g0742200 & Elongation factor EF-2 (Fragment) \\
\hline Os03g0565500 & Elongation factor $\mathrm{G} 1$, mitochondrial precursor (mEF-G-1) \\
\hline Os02g0456200 & G1 to S phase transition protein 1 homolog \\
\hline $\begin{array}{l}\text { Os01g0528000, } \\
\text { Os01g0652800 } \\
\text { Os01g0655400, } \\
\text { Os06g0688100 }\end{array}$ & Hypothetical protein \\
\hline $\begin{array}{l}\text { Os01g0229100, } \\
\text { Os02g0122300 }\end{array}$ & Conserved hypothetical protein \\
\hline Os02g0812400 & Nucleotidyl transferase domain containing protein \\
\hline Os05g0277300 & Peptide chain release factor 1 \\
\hline Os07g0503700 & Proteasome component region $\mathrm{PCl}$ domain containing protein \\
\hline Os01g0887200 & Winged helix DNA-binding domain containing protein \\
\hline Os06g0597400 & ZLL/PNH homologous protein \\
\hline Os04g0168100 & $\mathrm{Zn}$-finger, $\mathrm{C} 2 \mathrm{H} 2$ type domain containing protein \\
\hline
\end{tabular}

\section{(b) Negatively expressed transcripts (26)}

Os04g0533000 ATP-dependent RNA helicase p54 (Xp54)

Os05g0227700, Conserved hypothetical protein

Os07g0191700

Os03g0177400, EF-1 alpha

Os03g0178000

Os11g0116400 Elongation factor P (EF-P)
TABLE 7 | Continued

\begin{tabular}{|c|c|}
\hline Name & Annotation \\
\hline Os12g0541500 & Elongation factor Ts (EF-Ts) \\
\hline Os07g0614500 & Elongation factor 1-beta (EF-1-beta) \\
\hline $\begin{array}{l}\text { Os02g0220500, } \\
\text { Os02g0220600 }\end{array}$ & Elongation factor 1-gamma (EF-1-gamma) (eEF-1B gamma) \\
\hline Os02g0300700 & Eukaryotic translation initiation factor $1 \mathrm{~A}$ (EIF-1A) (EIF-4C) \\
\hline Os07g0681000 & $\begin{array}{l}\text { Eukaryotic translation initiation factor } 2 \text { beta subunit } \\
\text { (elF-2-beta) (P38) }\end{array}$ \\
\hline Os01g0120800 & $\begin{array}{l}\text { Eukaryotic translation initiation factor } 3 \text { subunit } 10 \text { (elF-3 } \\
\text { theta) }\end{array}$ \\
\hline Os07g0167000 & $\begin{array}{l}\text { Eukaryotic translation initiation factor } 3 \text { subunit } 6 \text { (elF-3 p48) } \\
\text { (elF3e) }\end{array}$ \\
\hline Os01g0970400 & $\begin{array}{l}\text { Eukaryotic translation initiation factor 4E-1 (elF4E-1) } \\
(\mathrm{elF}-4 \mathrm{E}-1)\end{array}$ \\
\hline Os12g0607100 & $\begin{array}{l}\text { Histone-lysine } \mathrm{N} \text {-methyltransferase, } \mathrm{H} 3 \text { lysine-9 specific (EC } \\
\text { 2.1.1.43) }\end{array}$ \\
\hline Os02g0794400 & Initiation factor three family protein \\
\hline Os05g0107700 & Transcription initiation factor IIA gamma chain (TFIIA-gamma) \\
\hline Os07g0639800 & Translation initiation factor IF6 family protein \\
\hline Os06g0338900 & Nucleotidyl transferase domain containing protein \\
\hline Os05g0277300 & Peptide chain release factor 1 \\
\hline Os02g0606100 & $\begin{array}{l}\text { Quinoprotein amine dehydrogenase, beta chain-like domain } \\
\text { containing protein }\end{array}$ \\
\hline Os02g0641800 & RNA helicase \\
\hline Os12g0165700 & $\begin{array}{l}\text { Transcription factors TFIIS, elongin A, CRSP70, conserved } \\
\text { domain containing protein }\end{array}$ \\
\hline $\begin{array}{l}\text { Os01g0772200, } \\
\text { Os10g0397200 }\end{array}$ & Winged helix DNA-binding domain containing protein \\
\hline
\end{tabular}

The chromosomal distribution of the positively expressed genes is shown in Figure S3.

growth, and development of plants (Tyrrell et al., 2007; Kim and Brandizzi, 2012). In this study, among the genes that are negatively expressed for shoot $\mathrm{Na} / \mathrm{K}$, six genes that significantly enriched the SNAP receptor activity in wide rice genotypes under salt stress were identified (Table S4a2). The bet like SNAREAtBS14a (Os02g0820700 and Os08g0563300) that were found to be significant was reported to control cell growth in Arabidopsis (Tai and Banfield, 2001). The syntaxin identified is AtSYP52 (encoded by Os02g0119400) was very recently described to act as t-SNARE when distributed in membrane TGN/PVC and plays a putative inhibitory role when present on the tonoplast in Arabidopsis (Benedictis et al., 2013). Another syntexin, OSM1/SYP61, was also reported to be involved in osmotic stress tolerance in Arabidopsis (Zhu et al., 2002). However, three other syntexins encoded by Os07g0164300, Os01g0254900, and Os06g0168500 that were found to be significant in this study might be novel syntexin and it would be of interest to know their specific role in future.

Chaperones are proteins involved in non-covalent folding or unfolding of other proteins and are believed to be expressed in response to high temperature and other cellular stresses. Yamada et al. (2002) identified a cytosolic chaperonin-containing TCP-1 $\alpha$ (CCT $\alpha)$ homolog that displayed enhanced salt tolerance in the mangrove plant, Bruguiera sexangula. In this study, six transcripts that significantly enriched chaperone binding 
activity under salt stress were identified. These are GrpE type 2 (Os08g0338700), GrpE protein family protein (Os04g0431100 and Os09g0284400), DRF2 (Os12g0456200) and one protein of unknown function (Os12g0456200) and another conserved hypothetical protein (Table S4a2).

\section{Interacting Network of Genes under Salt Stress}

The interactive networks analysis of all the significant genes (in total 578), as determined by SEA, revealed two networks (Figure 2). In the larger network, LOC_Os03g08050 (Os03g0177400) seemed to be the central protein which encodes for "Protein elongation factor (EF-1 alpha)." Most of the proteins in this network seem to be localized mainly in nucleus (blue), cytoplasm (pink), and mitochondria (light blue) and encode mainly for the translation factors such as LOC_Os02g56740 (protein translation initiation factor eIF-2B subunit epsilon, LOC_Os05g51500 (protein eukaryotic translation initiation factor 5B), LOC_Os07g44620 (protein eukaryotic translation initiation factor 6), LOC_Os05g41900 (protein translation initiation factor SUI1), LOC_Os02g19770 (protein eukaryotic translation initiation factor 1A), LOC_Os07g36940 (protein eukaryotic translation initiation factor $4 \mathrm{G}$ ) etc., and transcription factors such as LOC_Os06g14190 (protein NF-X1-type zinc finger protein). On the contrary, only a few proteins are located in the chloroplast (green) and plasma membrane (brown) and vacuole (yellow). The proteins localized in the chloroplast (green) are mainly catalytic proteins such as LOC_Os12g13390 (protein aspartyl aminopeptidase, putative), LOC_Os01g13190 (protein histidinol dehydrogenase, chloroplast precursor), LOC_Os02g10120 (protein lipoxygenase) and LOC_Os07g42180 (protein exportin 1). Proteins expressed in the vacuole includes LOC_Os06g23160 (protein bacterial transferase hexapeptide domain containing protein), LOC_Os01g12870 (protein eukaryotic translation initiation factor 3 subunit E-interacting protein), and LOC_Os02g39350 (protein eukaryotic translation initiation factor $2 \mathrm{~A}$ ) etc.

In the smaller network, all the proteins are SNAREs type proteins (SNAP receptor activity) with three localized in the vacuole encoding syntaxin (LOC_Os01g15110), vesicle transport v-SNARE protein (LOC_Os01g51120), and SNARE domain containing protein (LOC_Os02g02720) and one localized in the plasma membrane encoding vesicle transport v-SNARE protein (LOC_Os01g37980).

These finding probably points toward the hypothesis that in the wide natural gene pool, transcription and translation

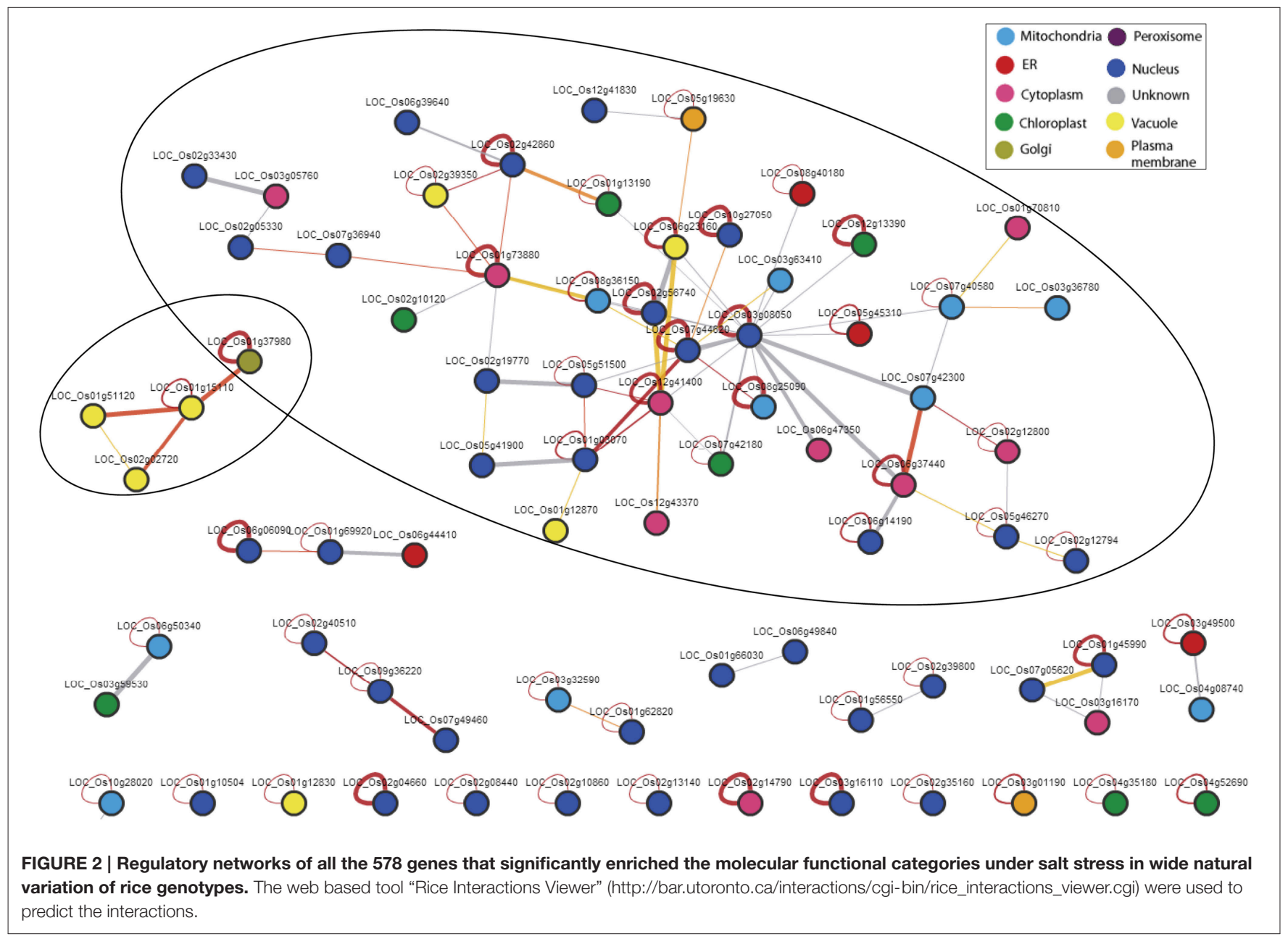


factor genes are the main regulators under salt stress and these genes are probably the initial defense response that coregulates in an interactive manner which ultimately cascades to induce the downstream genes that are essential for adaptation to environmental stresses.

\section{CONCLUSION}

The genes that are identified here provide a synergistic global salinity response picture representing the mechanisms that are active in the wide natural variation of rice genotypes which may not be referred back to individual genotypes in the context of this study (as gene expression responses of all the genotypes were analyzed together) but might be of interest to look at their specific role in individual rice genotypes in future. Besides, the gene expression profile of these genes in different cell or tissue types may also needed to be studied in a way to verify the pathways they are involved in via in situ analysis that will help to better understand their roles in salinity tolerance mechanism. Nonetheless, the products of these genes may hold the key to the evolutionary adaptive mechanism to cope with saline environments. Several of the identified genes were reported before either in rice or in other crop species, however, the novel genes and the genes with unknown function may enhance our understanding of stress adaptation once the role of these genes are functionally verified.

\section{AUTHOR CONTRIBUTIONS}

$\mathrm{MH}, \mathrm{GB}, \mathrm{JP}$, and BF jointly conceptualized and designed the project. $\mathrm{MH}$ has conducted all experiments, generated, and analyzed the data and prepared the draft script. GS helped in lab work, analysis and in finalizing the draft. JP, GB, and BL supervised the work, interpreted the results, and finalized the manuscript.

\section{ACKNOWLEDGMENTS}

This research and MRH was sponsored by The Commonwealth Scholarship Commission in the United Kingdom. GWB was supported by BBSRC grants BB/L010232/1, BB/J017604/1 and BB/N009754/1. We thank Dr. Glenn Gregorio and Flora de Guzman (IRRI) for providing the rice seeds and Dr. Gillian Kingston (Facility for Environmental Nanoscience Analysis and Characterization, the University of Birmingham, UK) for technical assistance with the ion analysis and the Genomic

\section{REFERENCES}

Achard, P., Cheng, H., De Grauwe, L., Decat, J., Schoutteten, H., Moritz, T., et al. (2006). Integration of plant responses to environmentally activated phytohormonal signals. Science 311, 91-94. doi: 10.1126/science.1118642

Aya, K., Suzuki, G., Suwabe, K., Hobo, T., Takahashi, H., Shiono, K., et al. (2011). Comprehensive network analysis of anther-expressed genes in rice by the combination of 33 laser microdissection and 143 spatiotemporal microarrays. PLoS ONE 6:e26162. doi: 10.1371/journal.pone.0026162
Services Facility team of the University of Birmingham, UK for their support during the entire processes of microarray experiment.

\section{SUPPLEMENTARY MATERIAL}

The Supplementary Material for this article can be found online at: http://journal.frontiersin.org/article/10.3389/fpls.2016. 00567

Figure S1 | The chromosomal distribution of the 107 positively expressed genes across the 12 chromosomes that significantly enriched the "signal transducer activity." The chromosome map is obtained by submitting the list of genes to the web based "Chromosome Map Tool" http://viewer.shigen.info/ oryzavw/maptool/MapTool.do (see Materials and Methods Section for details).

Figure S2 | The chromosomal distribution of the 81 negatively expressed genes across the 12 chromosomes that significantly enriched the "transcription factor activity."

Figure S3 | The chromosomal distribution of the 36 positively (A) and 26 negatively (B) expressed genes for shoot $\mathrm{Na} / \mathrm{K}$ that significantly enriched translation factor activity in wide natural rice genotypes under salt stress.

Table S1 | (a-n) Positively and negatively regulated significant probes for each of the 14 morpho-physiological traits in the eight rice genotypes representing wide natural variation in terms of salinity tolerance and genetic diversity under unstressed condition determined by Significance Analysis of Microarrays (SAM). Significant probes were selected based on the criteria of FDR $<5 \%$ ( $q$-value) and fold score $>2.0(d)$ and are presented along with the corresponding Locus ID and annotation.

Table S2 | (a-n) Positively and negatively regulated significant probes for each of the 14 morpho-physiological traits in the eight rice genotypes representing wide natural variation in terms of salinity tolerance and genetic diversity under stressed condition determined by Significance Analysis of Microarrays (SAM). Significant probes were selected based on the criteria of FDR $<5 \%$ ( $q$-value) and fold score $>2.0(d)$ and are presented along with the corresponding Locus ID and annotation.

Table S3 | Lists of probes along with the corresponding functional annotation and fold scores that commonly regulated for the trait Biomass under unstressed and stressed conditions in wide natural variation of rice genotypes.

Table S4 | Lists of significant GO categories of molecular functions and biological processes along with GO terms, $p$-values, FDR values, GO flash charts and schematic diagrams under unstressed condition for differentially regulated (positive and negative) genes for different morpho-physiological traits (determined by SEA analysis).

Table S5 | Lists of significant GO categories of molecular functions and biological processes along with GO terms, $p$-values, FDR values, GO flash charts and schematic diagrams under stressed condition for differentially regulated (positive and negative) genes for different morpho-physiological traits (determined by SEA analysis). 
drought resistance in rice. Plant Sci. 193, 8-17. doi: 10.1016/j.plantsci.2012. 05.003

Chen, Y. F., Li, L. Q., Xu, Q., Kong, Y. H., Wang, H., and Wu, W. H. (2009). The WRKY6 transcription factor modulates PHOSPHATE1 expression in response to low Pi stress in Arabidopsis. Plant Cell 21, 3554-3566. doi: 10.1105/tpc.108.064980

Cheng, Y., Qi, Y., Zhu, Q., Chen, X., Wang, N., Zhao, X., et al. (2009). New changes in the plasma-membrane-associated proteome of rice roots under salt stress. Proteomics 9, 3100-3114. doi: 10.1002/pmic.200800340

Clarke, B., and Rahman, S. (2005). A microarray analysis of wheat grain hardness. Theor. Appl. Genet. 110, 1259-1267. doi: 10.1007/s00122-005-1962-3

Close, T. J., and Wanamaker, S. I. (2004). A new resource for cereal genomics: 22K barley GeneChip comes of age. Plant Physiol. 134, 960-968. doi: 10.1104/pp.103.034462

Cotsaftis, O., Plett, D., Johnson, A. A. T., Walia, H., Wilson, C., Ismail, A. M., et al. (2011). Root-specific transcript profiling of contrasting rice genotypes in response to salinity stress. Mol. Plant 4, 25-41. doi: $10.1093 / \mathrm{mp} / \mathrm{ssq} 056$

Davidson, A. L., Dassa, E., Orelle, C., and Chen, J. (2008). Structure, function, and evolution of bacterial ATP-binding cassette systems. Microbiol. Mol. Biol. R. 72, 317-364. doi: 10.1128/MMBR.00031-07

Dong, J., Chen, C., and Chen, Z. (2003). Expression profiles of the Arabidopsis WRKY gene superfamily during plant defense response. Plant Mol. Biol. 51, 21-37. doi: 10.1023/A:1020780022549

Du, Z., Zhou, X., Ling, Y., Zhang, Z. H., and Su, Z. (2010). agriGO: a GO analysis toolkit for the agricultural community. Nucleic Acids Res. 38, W64-W70. doi: 10.1093/nar/gkq310

Duque, A. S., Almeida, A. M., De Silva, A. B., Da Silva, J. M., Farinha, A. P., Santos, D., et al. (2013). "Abiotic stress responses in plants: unraveling the complexity of genes and networks to survive," in Abiotic Stress - Plant Responses and Applications in Agriculture, eds K. Vahdati and C. Leslie (Rijeka: InTech), 3-23. doi: $10.5772 / 52779$

Echevarría-Zomeño, S., Yángüez, E., Fernández-Bautista, N., Castro-Sanz, A., Ferrando, A., and Castellano, M. (2013). Regulation of translation initiation under biotic and abiotic stresses. Int. J. Mol. Sci. 14, 4670-4683. doi: 10.3390/ijms14034670

Feder, M. E., and Walser, J. C. (2005). The biological limitations of transcriptomics in elucidating stress and stress responses. J. Evol. Biol. 18, 901-910. doi: 10.1111/j.1420-9101.2005.00921.x

Flowers, T. J., and Colmer, T. D. (2008). Salinity tolerance in halophytes. New Phytol. 179, 945-963. doi: 10.1111/j.1469-8137.2008.02531.x

Fujita, M., Fujita, Y., Noutoshi, Y., Takahashi, F., Narusaka, Y., YamaguchiShinozaki, K., et al. (2006). Crosstalk between abiotic and biotic stress responses: a current view from the points of convergence in the stress signaling networks. Curr. Opin. Plant Biol. 9, 436-442. doi: 10.1016/j.pbi.2006. 05.014

Goff, S. A., Ricke, D., Lan, T. H., Presting, G., Wang, R., Dunn, M., et al. (2002). A draft sequence of the rice genome (Oryza sativa L. ssp. Japonica). Science 296, 92-100. doi: 10.1126/science. 1068275

Horie, T., and Karahara, I. (2012). Salinity tolerance mechanisms in glycophytes: an overview with the central focus on rice plants. Rice 5:11. doi: 10.1186/19398433-5-11

Hossain, M. R., Pritchard, J., and Ford-Lloyd, B. V. (2015a). Qualitative and quantitative variation in the mechanisms of salinity tolerance determined by multivariate assessment of diverse rice (Oryza sativa L.) genotypes. Plant Genet. Resour. 1, 1-10. doi: 10.1017/S1479262115000118

Hossain, M. R., Vickers, L., Sharma, G., Livermore, T., Pritchrad, J., and FordLloyd, B. (2015b). "Salinity tolerance in plants: insights from transcriptomics studies," in Managing Salt Tolerance in Plants: Molecular and Genomic Perspectives, eds S. H. Wani and M. A. Hossain (CRC Press: Taylor \& Francis Group), 407-426.

Hossain, M. R. (2014). Salinity Tolerance and Transcriptomics in Rice. Ph.D. Thesis, University of Birmingham. Available online at: http://etheses.bham.ac.uk/5092/

Huang, D. W., Sherman, B. T., and Lempicki, R. A. (2009). Bioinformatics enrichment tools: paths toward the comprehensive functional analysis of large gene lists. Nucleic Acids Res. 37, 1-13. doi: 10.1093/nar/gkn923

Huang, G. T., Ma, S. L., Bai, L. P., Zhang, L., Ma, H., Jia, P., et al. (2012). Signal transduction during cold, salt, and drought stresses in plants. Mole. Biol. Rep. 39, 969-987. doi: 10.1007/s11033-011-0823-1
Jin, X. F., Xiong, A. S., Peng, R. H., Liu, J. G., Gao, F., Chen, J. M. et al. (2010). OsAREB1, an ABRE-binding protein responding to ABA and glucose, has multiple functions in Arabidopsis. BMB Rep. 43, 34-39. doi: 10.5483/BMBRep.2010.43.1.034

Kang, J., Hwang, J. U., Lee, M., Kim, Y. Y., Assmann, S. M., Martinoia, E., et al. (2010). PDR-type ABC transporter mediates cellular uptake of the phytohormone abscisic acid. Proc. Natl. Aca. Sci. U.S.A. 107, 2355-2360. doi: 10.1073/pnas.0909222107

Kasajima, I., Ide, Y., Hirai, M. Y., and Fujiwara, T. (2010). WRKY6 is involved in the response to boron deficiency in Arabidopsis thaliana. Physiol. Plant. 139, 80-92. doi: 10.1111/j.1399-3054.2010.01349.x

Kim, S. J., and Brandizzi, F. (2012). News and views into the SNARE complexity in Arabidopsis. Front. Plant. Sci. 3:28. doi: 10.3389/fpls.2012.00028

Kumari, M., Taylor, G. J., and Deyholos, M. K. (2008). Transcriptomic responses to aluminum stress in roots of Arabidopsis thaliana. Mol. Genet. Genom. 279, 339-357. doi: 10.1007/s10142-008-0088-5

Langridge, P., and Fleury, D. (2011). Making the most of 'omics' for crop breeding. Trends Biotechnol. 29, 33-40. doi: 10.1016/j.tibtech.2010.09.006

Li, S., Zhou, X., Chen, L., Huang, W., and Yu, D. (2010). Functional characterization of Arabidopsis thaliana WRKY39 in heat stress. Mol. Cells 29, 475-483. doi: 10.1007/s10059-010-0059-2

Lorenzo, L., Merchan, F., Laporte, P., Thompson, R., Clarke, J., Sousa, C., et al. (2009). A novel plant leucine-rich repeat receptor kinase regulates the response of Medicago truncatula roots to salt stress. Plant Cell 21, 668-680. doi: 10.1105/tpc.108.059576

Marshall, A., Aalen, R. B., Audenaert, D., Beeckman, T., Broadley, M. R., Butenko, M. A., et al. (2012). Tackling drought stress: receptor-Like kinases present new approaches. Plant Cell 24, 2262-2278. doi: 10.1105/tpc.112.096677

Martinoia, E., Klein, M., Geisler, M., Bovet, L., Forestier, C., Kolukisaoglu, U., et al. (2002). Multifunctionality of plant ABC transporters-more than just detoxifiers. Planta 214, 345-355. doi: 10.1007/s004250100661

Mochida, K., and Shinozaki, K. (2011). Advances in omics and bioinformatics tools for systems analyses of plant functions. Plant Cell Physiol. 52, 2017-2038. doi: $10.1093 / \mathrm{pcp} / \mathrm{pcr} 153$

Mukherjee, K., Choudhury, A., Gupta, B., Gupta, S., and Sengupta, D. (2006). An ABRE-binding factor, OSBZ8, is highly expressed in salt tolerant cultivars than in salt sensitive cultivars of Indica rice. BMC Plant Biol. 6:18. doi: 10.1186/1471-2229-6-18

Munns, R., James, R. A., and Lauchli, A. (2006). Approaches to increasing the salt tolerance of wheat and other cereals. J. Exp. Bot. 57, 1025-1043. doi: 10.1093/jxb/erj100

Munns, R., and Tester, M. (2008). Mechanisms of salinity tolerance. Annu. Rev. Plant Biol. 59, 651-681. doi: 10.1146/annurev.arplant.59.032607.092911

Muñoz, A., and Castellano, M. M. (2012). Regulation of translation initiation under abiotic stress conditions in plants: is it a conserved or not so conserved process among eukaryotes? Comp. Func. Genom. 2012:406357. doi: $10.1155 / 2012 / 406357$

Otsuki, L., Cheetham, S. W., and Brand, A. H. (2014). Freedom of expression: celltype-specific gene profiling. Wiley Interdiscip. Rev. Dev. Biol. 3, 429-443. doi: 10.1002/wdev.149

Ouyang, S. Q., Liu, Y. F., Liu, P., Lei, G., He, S. J., Ma, B., et al. (2010). Receptor-like kinase OsSIK1 improves drought and salt stress tolerance in rice (Oryza sativa) plants. Plant J. 62, 316-329. doi: 10.1111/j.1365-313X.2010.04146.x

Proietti, S., Bertini, L., Timperio, A. M., Zolla, L., Caporale, C., and Caruso, C. (2013). Crosstalk between salicylic acid and jasmonate in Arabidopsis investigated by an integrated proteomic and transcriptomic approach. Mol. Biosyst. 9, 1169-1187. doi: 10.1039/c3mb25569g

Rea, P. A. (1999). MRP subfamily ABC transporters from plants and yeast. J. Exp. Bot. 50, 895-913. doi: 10.1093/jxb

Senadheera, P., Singh, R. K., and Maathuis, F. J. M. (2009). Differentially expressed membrane transporters in rice roots may contribute to cultivar dependent salt tolerance. J. Exp. Bot. 60, 2553-2563. doi: 10.1093/jxb/erp099

Shelden, M. C., and Roessner, U. (2013). Advances in functional genomics for investigating salinity stress tolerance mechanisms in cereals. Front. Plant. Sci. 4:123. doi: $10.3389 /$ fpls. 2013.00123

Sinha, A. K., Jaggi, M., Raghuram, B., and Tuteja, N. (2011). Mitogen-activated protein kinase signaling in plants under abiotic stress. Plant Signal. Behav. 6, 196-203. doi: 10.4161/psb.6.2.14701 
Sreenivasulu, N., and Miranda, M. (2004). Transcriptome changes in foxtail millet genotypes at high salinity: identification and characterization of a PHGPX gene specifically up-regulated by $\mathrm{NaCl}$ in a salt-tolerant line. J. Plant Physiol. 161, 467-477. doi: 10.1078/0176-1617-01112

Tai, W. C., and Banfield, D. K. (2001). AtBS14a and AtBS14b, two Bet1/Sft1like SNAREs from Arabidopsis thaliana that complement mutations in the yeast SFT1 gene. FEBS Lett. 500, 177-182. doi: 10.1016/S0014-5793(01) 02599-6

Tanaka, H., Osakabe, Y., Katsura, S., Mizuno, S., Maruyama, K., Kusakabe, K., et al. (2012). Abiotic stress-inducible receptor-like kinases negatively control ABA signaling in Arabidopsis. Plant J. 70, 599-613. doi: 10.1111/j.1365313X.2012.04901.X

Tang, N., Zhang, H., Li, X. H., Xiao, J. H., and Xiong, L. Z. (2012). Constitutive activation of transcription factor OsbZIP46 improves drought tolerance in rice. Plant Physiol. 158, 1755-1768. doi: 10.1104/pp.111

Taylor-Teeples, M., Ron, M., and Brady, S. M. (2011). Novel biological insights revealed from cell type-specific expression profiling. Curr. Opin. Plant Biol. 14, 601-607. doi: 10.1016/j.pbi.2011.05.007

Tusher, V. G., Tibshirani, R., and Chu, G. (2001). Significance analysis of microarrays applied to the ionizing radiation response. Proc. Natl. Acad. Sci. U.S.A. 98, 5116-5121. doi: 10.1073/pnas.091062498

Tyrrell, M., Campanoni, P., Sutter, J. U., Pratelli, R., Paneque, M., Sokolovski, S., et al. (2007). Selective targeting of plasma membrane and tonoplast traffic by inhibitory (dominant-negative) SNARE fragments. Plant J. 51, 1099-1115. doi: 10.1111/j.1365-313X.2007.03206.x

Ueda, A., Kathiresan, A., Bennett, J., and Takabe, T. (2006). Comparative transcriptome analyses of barley and rice under salt stress. Theor. Appl. Genet. 112, 1286-1294. doi: 10.1007/s00122-006-0231-4

Walia, H., and Wilson, C. (2007). Genome-wide transcriptional analysis of salinity stressed Japonica and Indica rice genotypes during panicle initiation stage. Plant Mol. Biol. 63, 609-623. doi: 10.1007/s11103-006-9112-0

Walia, H., and Wilson, C. (2009). Comparing genomic expression patterns across plant species reveals highly diverged transcriptional dynamics in response to salt stress. BMC Genomics 10:398. doi: 10.1104/pp.105. 065961

Walia, H., Wilson, C., Condamine, P., Liu, X., Ismail, A. M., Zeng, L., et al. (2005). Comparative transcriptional profiling of two contrasting rice genotypes under salinity stress during the vegetative growth stage. Plant Physiol. 139, 822-835. doi: 10.1104/pp.105.065961

Wang, H., Miyazaki, S., Kawai, K., Deyholos, M., Galbraith, D. W., and Bohnert, H. J. (2003). Temporal progression of gene expression responses to salt shock in maize roots. Plant. Mol. Biol. 52, 873-891. doi: 10.1023/A:1025029026375
Wei, T., Ou, B., Li, J., Zhao, Y., Guo, D., Zhu, Y., et al. (2013). Transcriptional profiling of rice early response to magnaporthe oryzae identified OsWRKYs as important regulators in rice blast resistance. PLOS ONE 8:e59720. doi: 10.1371/journal.pone.0059720

Wek, R. C., Jiang, H. Y., and Anthony, T. G. (2006). Coping with stress: eIF2 kinases and translational control. Biochem. Soc. Trans. 34, 7-11. doi: 10.1042/BST0340007

Wu, D., Cai, S., Chen, M., Ye, L., Chen, Z., Zhang, H., et al. (2013). Tissue metabolic responses to salt stress in wild and cultivated barley. PLOS ONE 8:e55431. doi: 10.1371/journal.pone.0055431

Xiang, Y., Tang, N., Du, H., Ye, H. Y., and Xiong, L. Z. (2008). Characterization of OsbZIP23 as a key player of the basic leucine zipper transcription factor family for conferring abscisic acid sensitivity and salinity and drought tolerance in rice. Plant Physiol. 148, 1938-1952. doi: 10. 1104/pp.108.128199

Yamada, A., Sekiguchi, M., Mimura, T., and Ozeki, Y. (2002). The role of plant CCT $\alpha$ in salt- and osmotic-stress tolerance. Plant Cell Physiol. 43, 1043-1048. doi: $10.1093 / \mathrm{pcp} / \mathrm{pcf} 120$

Zhao, J., Sun, Z., Zheng, J., Guo, X., Dong, Z., Huai, J., et al. (2009). Cloning and characterization of a novel CBL-interacting protein kinase from maize. Plant Mol. Biol. 69, 661-674. doi: 10.1007/s11103-008-9445-y

Zhu, J., Gong, Z., Zhang, C., Song, C. P., Damsz, B., Inan, G., et al. (2002). OSM1/SYP61: a syntaxin protein in Arabidopsis controls abscisic acidmediated and non-abscisic acid-mediated responses to abiotic stress. Plant Cell 14, 3009-3028. doi: 10.1093/jxb/erq204

Zou, C. S., Jiang, W. B., and Yu, D. Q. (2010). Male gametophyte-specific WRKY34 transcription factor mediates cold sensitivity of mature pollen in Arabidopsis. J. Exp.Bot. 61, 3901-3914. doi: 10.1093/jxb/erq204

Zou, M. J., Guan, Y. C., Ren, H. B., Zhang, F., and Chen, F. (2008). A bZIP transcription factor, OsABI5, is involved in rice fertility and stress tolerance. Plant Mol. Biol. 66, 675-683. doi: 10.1093/jxb/erq204

Conflict of Interest Statement: The authors declare that the research was conducted in the absence of any commercial or financial relationships that could be construed as a potential conflict of interest.

Copyright (c) 2016 Hossain, Bassel, Pritchard, Sharma and Ford-Lloyd. This is an open-access article distributed under the terms of the Creative Commons Attribution License (CC BY). The use, distribution or reproduction in other forums is permitted, provided the original author(s) or licensor are credited and that the original publication in this journal is cited, in accordance with accepted academic practice. No use, distribution or reproduction is permitted which does not comply with these terms. 